\title{
The complete chloroplast genome sequence of the leafy bladderwort, Utricularia foliosa L. (Lentibulariaceae)
}

\author{
Saura R. Silva ${ }^{1} \cdot$ Daniel G. Pinheiro $^{2} \cdot$ Elliott J. Meer $^{3} \cdot$ Todd P. Michael $^{3}$ • \\ Alessandro M. Varani ${ }^{2} \cdot$ Vitor F. O. Miranda ${ }^{1,4}$
}

Received: 28 October 2016 / Accepted: 15 November 2016 / Published online: 18 November 2016

(C) Springer Science+Business Media Dordrecht 2016

\begin{abstract}
Utricularia foliosa L., commonly known as leafy bladderwort, is a widespread aquatic carnivorous plant from the Lentibulariaceae family. The species of the Utricularia and Genlisea genera are known to possess the smallest nuclear genomes across angiosperms. This study reveals that $U$. foliosa have a typical chloroplast genome of $150,851 \mathrm{bp}$ in length, comprised of a large single-copy region (LSC) of $82,720 \mathrm{bp}$, a small single-copy region (SSC) of 17,481 bp, and inverted repeat regions (IRs) of $25,325 \mathrm{bp}$. A total of 139 genes, 88 of which are protein coding genes, 37 tRNA genes, eight rRNA genes and six pseudogenes were identified. All plastid NAD(P)H-dehydrogenase genes are present as intact copies. Phylogenetic analyses indicate that $U$. foliosa is closely related to other suspended or affixed aquatic species belonging to the Utricularia sect. Utricularia.
\end{abstract}

Keywords Carnivorous plant $\cdot$ Lentibulariaceae . Bladderwort $\cdot$ Plastome $\cdot$ CpDNA

Alessandro M. Varani

amvarani@fcav.unesp.br

$\triangle$ Vitor F. O. Miranda

vmiranda@fcav.unesp.br

1 Instituto de Biociências, UNESP - Univ Estadual Paulista, Câmpus Botucatu, São Paulo, Brazil

2 Departamento de Tecnologia, Faculdade de Ciências Agrárias e Veterinárias, UNESP - Univ Estadual Paulista, Câmpus Jaboticabal, São Paulo, Brazil

3 Ibis Bioscience, Computational Genomics, Carlsbad, CA, USA

4 Departamento de Biologia Aplicada à Agropecuária, Faculdade de Ciências Agrárias e Veterinárias, UNESP Univ Estadual Paulista, Câmpus Jaboticabal, São Paulo, Brazil
Utricularia foliosa L. (leafy bladderwort, Lentibulariaceae) is a perennial free floating aquatic plant (Taylor 1989) that grows in lakes, ponds and swamps. The genus Utricularia, which also includes Genlisea, has increasingly gained researchers attention due to the dynamic size of its nuclear DNA content (Albert et al. 2010; Veleba et al. 2014). Despite its widespread distribution in the Americas, Africa and Madagascar, only a few studies of $U$. foliosa have been conducted. These studies have focused mainly on prey composition (Solís-Parra and Críales-Hernández 2016), bladder respiration and photosynthesis (Adamec 2006). Only a single phylogenetic analysis, with one molecular marker, has been used to determine its phylogenetic position within the Utricularia genus (Silva et al. 2016). U. foliosa can bloom throughout the year, and is found in slowly flowing, shallow to deep water bodies, such as lakes, marshes and rivers (Taylor 1989). These habitats, where U. foliosa is commonly found, are suffering rapid environmental loss due to negative anthropic impacts such as drainage and conversion to urban or agricultural activities.

Herein, we report the first complete chloroplast genome of $U$. foliosa. The annotated U. foliosa genome has been deposited in the public database, GenBank, with the accession number: KY025562 (BioProject: PRJNA350159; BioSample: SAMN05933770).

Total genomic DNA was extracted from silica gel dried inflorescences of Utricularia foliosa, collected from ponds in the Tietê River in Mogi das Cruzes (São Paulo, Brazil; lat. -23.532294 , long. $-46.202648,765 \mathrm{~m}$ a.s.l.) using a modified CTAB method (Doyle and Doyle 1987). Herbarium voucher (V.F.O.de Miranda et al. 2070) is deposited at the JABU Herbarium at Universidade Estadual Paulista (UNESP/ FCAV).

Genomic sequencing was performed on the Illumina MiSeq Platform (Illumina, San Diego, CA), resulting in 


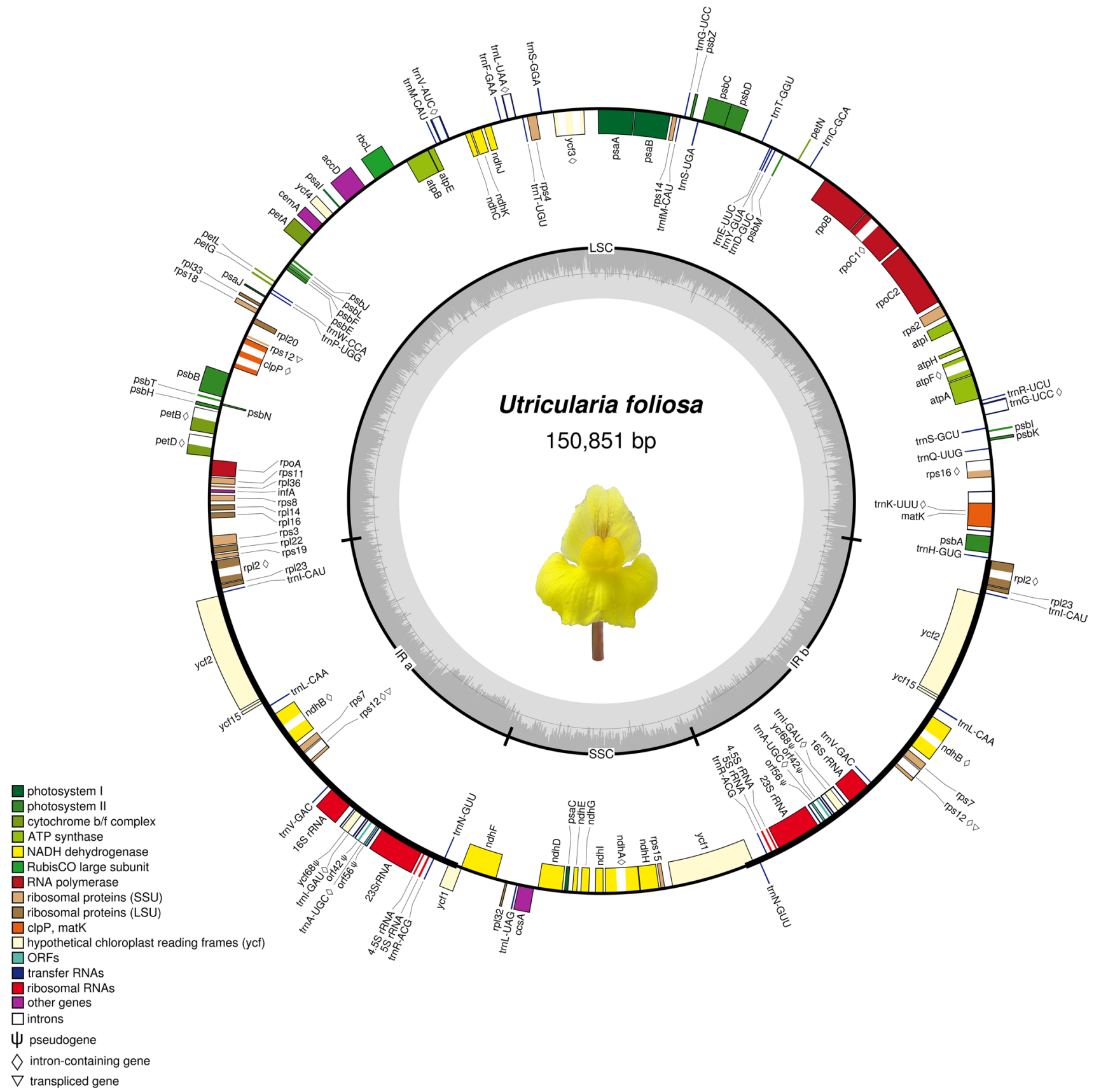

Fig. 1 Gene map of the Utricularia foliosa chloroplast genome. Genes belonging to different functional groups are shown in different colors. The open diamond and chi indicate genes with intron(s) and pseudogene, respectively. (Color figure online)

2,933,837 paired-end reads $(2 \times 300 \mathrm{bp})$. The reads were quality trimmed using Platanus_trim v1.0.7 (Kajitani et al. 2014) and aligned to the reference Utricularia gibba chloroplast genome (Genbank accession number: NC_021449) using Bowtie 2 v.2.2.3 (Langmead and Salzberg 2012). The matched chloroplast reads (135,879 reads) were de novo assembled with SPAdes 3.9 (Bankevich et al. 2012) and ambiguous regions were picked out to extend the length using an iteration method with MITObim v.1.8 (Hahn et al. 2013). Quality filtered reads were mapped back to the chloroplast genome using Bowtie2 (98.95\% overall alignment rate) to confirm assembly accuracy quality and repeat region junctions. The gene annotation was performed using DOGMA (http://evogen.jgi-psf.org/dogma/) (Wyman et al. 2004) and confirmed by BLASTn searches on NCBI nucleotide collection database (nt). tRNA genes were annotated using DOGMA and ARAGORN v1.2 (Laslett and Canback 2004). The genome map was generated using OGDRAW 


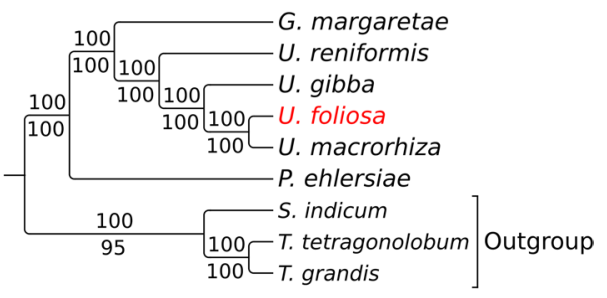

Fig. 2 Bayesian Inference phylogram based on 53 genes of complete chloroplast genome sequences deposited in public databases. Numbers below the nodes are bootstrap values for maximum likelihood analysis and above the nodes are posterior probability values. Accession numbers: Genlisea margaretae NC_025652, Pinguicula ehlersiae NC_023463, Sesamum indicum JN_637766, Tanaecium tetragonolobum NC_027955, Tectona grandis NC_020098, Utricularia gibba NC_021449, U. reniformis NC_029719, U. macrorhiza NC_025653

v. 1.2 (http://ogdraw.mpimp-golm.mpg.de/) (Lohse et al. 2013) followed by manual modifications.

The phylogenetic position of $U$. foliosa was inferred using 53 genes of the previously published Lentibulariaceae chloroplast genomes, with the Tectona grandis (Lamiaceae), Sesamum indicum (Pedaliaceae) and Tanaecium tetragonolobum (Bignoniaceae) plastomes used as outgroups.

The sequences were aligned using MAFFT v.7 (Katoh and Standley 2013) and probabilistic phylogeny was conducted using Mr. Bayes v.3 for Bayesian inference (Ronquist and Huelsenbeck 2003) with $5 \times 10^{5}$ generations, using the default parameters, and RAxML for maximum likelihood (Stamatakis 2014) using the default parameters with bootstrap support of $10^{4}$ pseudoreplicates. The evolutionary model used was GTR $+\mathrm{G}$, tested a priori using jModelTest (Darriba et al. 2012).

The chloroplast genome of Utricularia foliosa is a typical quadripartite structure with a length of $150,851 \mathrm{bp}$, which contained inverted repeats (IR) of 25,325 bp separated by a large single-copy (LSC) and a small singlecopy (SSC) of $82,720 \mathrm{bp}$ and $17,481 \mathrm{bp}$, respectively. The cpDNA contains 139 genes, comprising 88 protein-coding genes, 8 ribosomal RNA genes, 37 tRNA genes and 6 pseudogenes. Among the annotated genes, 15 of them contain one intron $(a t p \mathrm{~F}, \operatorname{pet} \mathrm{B}, \operatorname{pet} \mathrm{D}, r p l 2, r p l 16, r p o \mathrm{C} 1, r p s 12$,

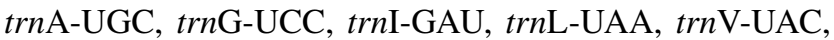
$\operatorname{tr} n \mathrm{~K}-\mathrm{UUU}, n d h \mathrm{~B}, n d h \mathrm{~A})$, and $y c f 3$ and $c l p \mathrm{P}$ genes contain two introns. The overall GC content of $U$. foliosa cp genome is $37.32 \%$, while it was $43 \%$ for IRs, $35.20 \%$ for LSC and $30.83 \%$ for SSC regions (Fig. 1). Interestingly, all the eleven subunits of the plastid NAD $(\mathrm{P}) \mathrm{H}$-dehydrogenase (ndh) genes are present as intact copies, corroborating previous studies which suggested that the plastid ndh complete gene set may exist only in aquatic forms of carnivorous plants in the Lentibulariaceae family (Silva et al. 2016).
Previous phylogenetic studies have shown that Lentibulariaceae and Utricularia are monophyletic groups, supported by molecular and morphological characteristics (Müller et al. 2004; Silva et al. 2016). Our phylogenetic analysis strongly supports the positioning of $U$. foliosa nested within the known related aquatic species $U$. gibba and U. macrorhiza (Utricularia sect. Utricularia) (Fig. 2).

Acknowledgements The first author was supported by the Coordenação de Aperfeiçoamento de Pessoal de Nível Superior (CAPES). VFOM thanks for the Conselho Nacional de Desenvolvimento Científico e Tecnológico (CNPq) for the fellowship (Bolsa de Produtividade-Proc. \# 309040/2014-0).

\section{References}

Adamec L (2006) Respiration and photosynthesis of bladders and leaves of aquatic Utricularia species. Plant Biol 8:765-769. doi: 10.1055/s-2006-924540

Albert VA, Jobson RW, Michael TP, Taylor DJ (2010) The carnivorous bladderwort (Utricularia, Lentibulariaceae): a system inflates. J Exp Bot 61:5-9. doi:10.1093/jxb/erp349

Bankevich A, Nurk S, Antipov D, Gurevich AA, Dvorkin M, Kulikov AS, Lesin VM, Nikolenko SI, Pham S, Prjibelski AD (2012) SPAdes: a new genome assembly algorithm and its applications to single-cell sequencing. J Comput Biol 19:455-477. doi:10.1089/cmb.2012.0021

Darriba D, Taboada GL, Doallo R, Posada D (2012) jModelTest 2: more models, new heuristics and parallel computing. Nat Methods 9:772. doi:10.1038/nmeth.2109

Doyle JJ, Doyle JL (1987) A rapid DNA isolation procedure for small quantities of fresh leaf tissue. Phytochem Bull 19:11-15

Hahn C, Bachmann L, Chevreux B (2013) Reconstructing mitochondrial genomes directly from genomic next-generation sequencing reads: a baiting and iterative mapping approach. Nucleic Acids Res 41(13):e129. doi:10.1093/nar/gkt371

Kajitani R, Toshimoto K, Noguchi H, Toyoda A, Ogura Y, Okuno M, Yabana M, Harada M, Nagayasu E, Maruyama H, Kohara Y, Fujiyama A, Hayashi T, Itoh T (2014) "Efficient de novo assembly of highly heterozygous genomes from whole-genome shotgun short reads". Genome Res 24(8):1384-1395. doi:10.1101/ gr.170720.113

Katoh K, Standley DM (2013) MAFFT multiple sequence alignment software version 7: improvements in performance and usability. Mol Biol Evol 30:772-780. doi:10.1093/molbev/mst010

Langmead B, Salzberg SL (2012) Fast gapped-read alignment with Bowtie 2. Nat Methods 9:357-359. doi:10.1038/nmeth.1923

Laslett D, Canback B (2004) ARAGORN, a program to detect tRNA genes and tmRNA genes in nucleotide sequences. Nucleic Acids Res 32:11-16. doi:10.1093/nar/gkh152

Lohse M, Drechsel O, Kahlau S, Bock R (2013) OrganellarGenomeDRAW-a suite of tools for generating physical maps of plastid and mitochondrial genomes and visualizing expression data sets. Nucleic Acids Res 41:575-581. doi:10.1093/nar/gkt289

Müller K, Borsch T, Legendre L, Porembski S, Theisen I, Barthlott W (2004) Evolution of carnivory in Lentibulariaceae and the Lamiales. Plant Biol 6:1-14. doi:10.1055/s-2004-817909

Ronquist F, Huelsenbeck JP (2003) MRBAYES 3: Bayesian phylogenetic inference under mixed models. Bioinformatics 19:15721574. doi:10.1093/bioinformatics/btg 180

Silva SR, Diaz YCA, Penha HA, Pinheiro DG, Fernandes CC, Miranda VFO, Michael TP, Varani, AM (2016) The chloroplast 
genome of Utricularia reniformis sheds light on the evolution of the ndh gene complex of terrestrial carnivorous plants from the Lentibulariaceae family. Plos ONE 11(10):e0165176. doi:10.1371/journal.pone.0165176

Solís-Parra J, Críales-Hernández M (2016) Capture and selectivity of zooplankton by Utricularia foliosa (Lentibulariaceae) in the Ciénaga de Paredes, Santander, Colombia. J Trop Biol Cons 64:1297-1310. doi:10.15517/rbt.v64i3.21387

Stamatakis A (2014) RAxML version 8: a tool for phylogenetic analysis and post-analysis of large phylogenies. Bioinformatics 30:1312-1313. doi:10.1093/bioinformatics/btu033
Taylor P (1989) The genus Utricularia: a taxonomic monograph. Kew bulletin additional series XIV. Kew Royal Botanic Gardens, London

Veleba A, Bureš P, Adamec L, Šmarda P, Lipnerová I, Horová L (2014) Genome size and genomic GC content evolution in the miniature genome-sized family Lentibulariaceae. New Phytol 203:22-28. doi:10.1111/nph.12790

Wyman SK, Jansen RK, Boore JL (2004) Automatic annotation of organellar genomes with DOGMA. Bioinformatics 20:32523255. doi:10.1093/bioinformatics/bth352 